\title{
A Study Of Prevalence Of Different Species Of Malassezia Causing Pityriasis Versicolor In Different Age Groups In A Tertiary Care Hospital In Kolkata
}

\author{
${ }^{1}$ Sampurna Biswas Pramanik, ${ }^{2}$ Manas Bandopadhyay, ${ }^{3}$ Anita Nandi (Mitra). \\ ${ }^{1)}$ Demonstrator, Dept. Of Microbiology, Medical College\& Hospital,Kolkata \\ ${ }^{2)}$ Professor, Dept. Of Microbiology,R. G. Kar Medical College \& Hospital,Kolkata \\ ${ }^{3}$ Demonstrator, Dept. Of Microbiology, Medical College\& Hospital,Kolkata
}

\begin{abstract}
Pityriasis versicolor is a superficial skin disease involving upto the stratum corneum layer of the skin and the sebaceous glands. The main complain of the patients being cosmetic and itchiness on profuse sweating.Most common causative agent is Malassezia species.Materials and Methods- Skin scrapings were collected from 88 consecutive patients from Dermatology OPD, RGKMCH of various age groups.Speciation done in Microbiology laboratory..Direct smear preparation with $10 \% \mathrm{KOH}$ and culture inoculation primarily in SDCA and MDA was done, followed by LCB mount and biochemical tests for speciation. Observation: In the present study, higher incidence was found among 15-25 years age group (81.81\%) and the most common species being Malassezia furfur.Conclusion: Ptyriasis versicolor is most prevalent in the age group of 15-25 yrs and M.furfur to be the most prevalent species, followed by M. globosa, M. restricta and M. sympodialis.
\end{abstract}

Key words: Age groups, Malassezia sp,Pityriasis versicolor.

\section{Introduction}

Pityriasis versicolor is a mild, chronic infection of the skin caused by Malassezia yeasts and characterized by discrete or confluent, scaly, discoloured or depigmented areas, mainly on the upper trunk[1]. It is a superficial cutaneous fungal infection of the stratum corneum[2]. The lesions are characterized by a branny or furfuraceous consistency. They are discrete or concrescent and appear as discoloured or depigmented areas of the skin. Margins of the macules are sharply delineated. Lesions tend to be discrete in the beginning, but may fuse to give a serpiginous border as they enlarge. Hair shafts and nails are not infected. Hyperpigmentation with fawn-coloured macules is much more common than hypopigmentation. A light scaling may be seen .

The lesions start as tiny, multiple, macular spots that soon scale and enlarge. The lesions have often been present for more than a year when the patient is seen by a physician. Patients who seek medical attention do so for cosmetic reasons, but upto a third will have noted slight itching or burning in the area of the lesions, particularly when sweating. The expression of infection is promoted by heat and humidity. Left untreated, the infection waxes and wanes for months and years.

A common observation in reviewing a series of cases of pityriasis versicolor is that both hypo and hyperpigmented lesions occur, sometimes on the same patient.

Members of the genus Malassezia are opportunistic yeasts of increasing importance, due in large part to advances in detection and culture methodology which have been allowed their investigation and revealed their importance in human and animal diseases[3,4]. The lipid- dependent genus Malassezia is considered as the causative organism. Of which M.furfur, M.globosa, M. sympodialis, M. slooffiae, M. obtusa are considered to be the most common aetiologic agents. M. furfur responsible for pityriasis vericolor mainly under tropical climate [5]. The differences in frequencies of Malassezia species among different studies may be attributed to different culture media (MDA / LNA) and perhaps to ethnic and geographic factors [6].

The role of sex in propensity to development of PV is still unclear. Some found that PV is more common in men, while others indicated higher incidence of infection in females which may be due to extra attention to beauty and skin hygiene [7,8]. Age prevalence to be 20-30yrs. Although the morphologic characteristics (colony \& microscopic examination) for Malassezia is used for primary identification,but they don't provide sufficient information for specific identification of isolates.So,to avoid confusion, physiological tests should be carried out. Two simple preliminary tests for this purpose are, the subculture on $\operatorname{SDA}\left(\right.$ at $\left.31{ }^{\circ} \mathrm{C}\right)$ and the catalase reaction. All lipid-dependent species except $\mathrm{M}$. restricta exhibit a catalase positive reaction. 


\section{Materials \& Methods:}

The study was conducted at the department of Microbiology in collaboration with the Department of Dermatology, R.G.Kar Medical College \& Hospital,(RGKMCH)Kolkata-700004, during the period of July 2011 to June 2012.Patients from Kolkata and adjoining districts attending Dermatology OPD with hypo- or hyper-pigmented macular lesions of different age groups. Skin scrapings were collected from 88 consecutive patients from Dermatology OPD, RGKMCH. The study was prospective, cross-sectional, tertiary care hospital based. For each patient following protocol was followed:

\section{Clinical Assessment:}

Patients attending Dermatology OPD, RGKMCH with hypo/hyperpigmented macular lesions during July 2011 to June 2012 were examined for pityriasis versicolor. Detailed history regarding socioeconomic condition, duration of the illness, associated itchiness were taken. Any drug intake history was also enquired about.

\section{Specimen Collection:}

Skin scrapings collected from the junction of active lesion and healthy and normal skin with the help of cellotape and sterile scalpel blade.

\section{Sample Processing:}

All the samples were examined by $10 \% \mathrm{KOH}$ under direct microscopy. Three skin samples were inoculated in SDCA with olive oil overlay,MDA and BA.Cultures were incubated at $37{ }^{\circ} \mathrm{C}$ and $25^{\circ} \mathrm{C}$ on D3/D7 for any fungal growth. Fungal growth further processed by gram staining, LCB mount and biochemical tests for species identification such as catalase and urease test, glycine assimilation and tween $(20,40,60,80)$ utilization.

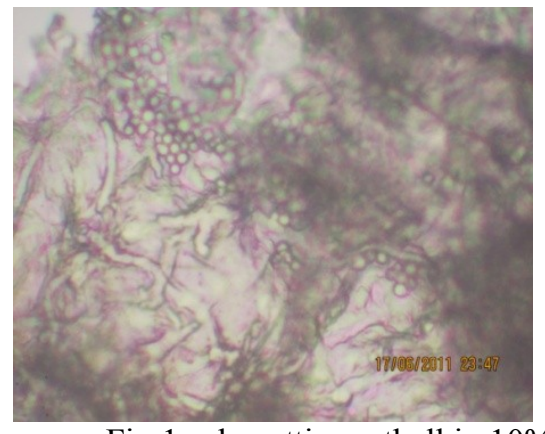

Fig 1:sphagetti-meatball in $10 \% \mathrm{KOH}$.

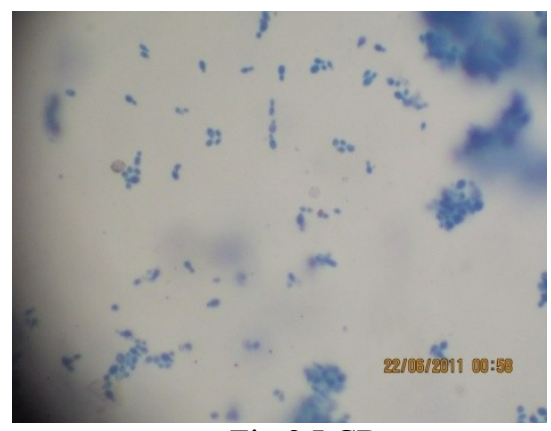

Fig 2:LCB mount

\section{Results:}

The study was carried out in the Department of Microbiology in collaboration with the Department of Dermatology, R.G.Kar Medical College and Hospital.

Cases included both hypo- and hyper- pigmented macular rashes, clinically suspected as pityriasis versicolor. Samples were collected from 98 individuals attending Dermatology OPD.

Out of 98 clinically suspected cases, 88 were found to be suggestive for pityriasis versicolor all of which also showed growth of Malassezia on cultivation. Remaining 10 did not show any positive finding either in $\mathrm{KOH}$ preparation or culture.

Table I:Distribution Of Cases According To Age Groups:

\begin{tabular}{|l|l|l|l|}
\hline $\begin{array}{l}\text { TOTAL NO } \\
\text { OF CASES }\end{array}$ & $<15$ YRS & $15-25$ YRS & $>25 Y R S$ \\
\hline 88 & $2(2.27 \%)$ & $72(81.81 \%)$ & $14(15.90 \%)$ \\
\hline
\end{tabular}

Table 1 showing maximum prevalence of the disease in the age group of $15-25$ yrs $(81.81 \%)$. 


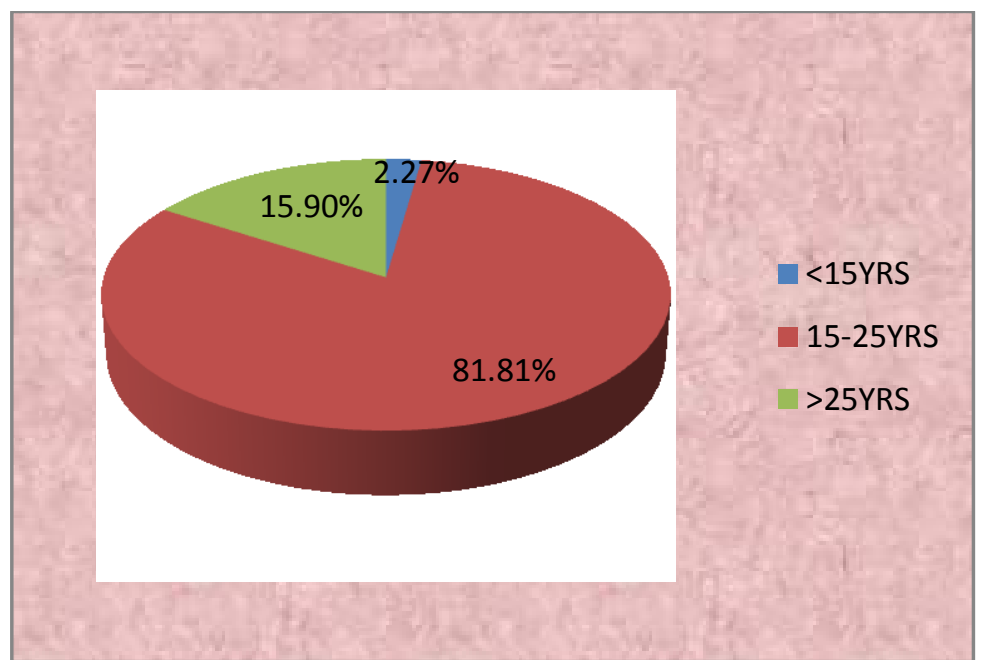

Fig 3: Pie Diagram Showing Age Group Wise Distribution Of Cases

Table Ii: Prevalence Of Different Malassezia Species Among The Total Culture Positive Cases:

\begin{tabular}{|l|l|}
\hline TOTAL NO. OF CULTURE POSITIVE CASES & 88 \\
\hline M. furfur & $53(60.23 \%)$ \\
M. globosa & $25(28.41 \%)$ \\
M. restricta & $6(6.82 \%)$ \\
M. sympodialis & $4(4.55 \%)$ \\
\hline
\end{tabular}

Table 2 demonstrating the prevalence of four malassezia species among 88 culture positive isolates. M. furfur isolates are highest (60.23\%) in number followed by M.globosa (28.41\%), M. restricta (28.41\%) and M.sympodialis (4.55\%).This configuration is also depicted through a pie diagram.

Table Iii: Prevalence Of Malassezia Species According To The Age Groups:

\begin{tabular}{|l|l|l|l|l|}
\hline SPECIES & $<15$ YRS & $15-25 Y R S$ & $>25 Y R S$ & TOTAL \\
\hline M. furfur & $1(1.14 \%)$ & $44(50 \%)$ & $\mathbf{8}(9.09 \%)$ & $53(60.23 \%)$ \\
M. globosa & $1(1.14 \%)$ & $20(22.73 \%)$ & $4(4.55 \%)$ & $25(28.41 \%)$ \\
M. restricta & $0(0 \%)$ & $5(5.68 \%)$ & $1(1.14 \%)$ & $6(6.82 \%)$ \\
M. sympodialis & $0(0 \%)$ & $3(3.41 \%)$ & $1(1.14 \%)$ & $4(4.55 \%)$ \\
TOTAL(88) & $2(2.27 \%)$ & $72(81.82 \%)$ & $14(15.91 \%)$ & $(88)$ \\
\hline
\end{tabular}

Table 3 showing higher prevalence of M.furfur (60.23\%) among the 15 -25yrs age group (50\%), next to it was M. globosa which accounted for 20 isolates $(22.73 \%)$ among the total 88 cases. This table also shows no incidence of M.restricta and M.sympodialis in below $15 \mathrm{yrs}$ age group(2.27\%) and very low incidence (1.14\% each) among above $25 \mathrm{yrs}$ population.

\section{Discussion:}

Pityriasis versicolor is a mild, chronic infection of skin caused by malassezia yeasts and characterized by discrete confluent, scaly, discoloured or depigmented areas, mainly on the upper trunk. Colonization by these species is specially dense in the scalp, the upper trunk and flexures, areas rich in sebaceous glands and their secretions [1]. The characteristic changes include hyperkeratosis, parakeratosis, acanthosis and a mild inflammatory infiltrate. The organism can be seen in the upper layers of the stratum corneum and electron microscopy shows the presence of organisms intercellularly. Increased cell turnover is found in affected cells .

The study was conducted on patients attending Dermatology OPD. The study was carried out in the department of Microbiology in collaboration with the Dermatology department. R.G.Kar Medical College and Hospital, during the midway of July, 2011 to June, 2012.

Both hypo- and hyper-pigmented macular rashes, clinically suspected as pityriasis versicolor were included in the study population. Samples were collected from 98 individuals attending Dermatology OPD. Out of 98 clinically suspected cases, 88 were found to be suggestive for pityriasis versicolor, all of which also showed growth of malassezia on cultivation. Remaining 10 did not show any positive finding either in $\mathrm{KOH}$ preparation or culture.

Pityriasis versicolor is known to affect mainly young adults $[9,10,11]$. The commonest age group affected is 25-30 years in males and 20-25years in females [12] but it may occur in children[12,13,14] and in the aged. Though cases occurring in 11 month and 12 month old and less than 
6 month old children have also been reported[15]. The youngest case of PV was a 8 week old male infant[16]. In the present study, higher incidence was found among 15-25 years age group (81.81\%) and age group below 15years lead to the least incidence (2.27\%). This was in accordance with the findings of other studies [17]. A study done on Central African population also showed results similar to that of the present study. Another study conducted in Northern Iran showed highest prevalence in 20-25 years age group[18]. This may be due to varying geographical and environmental conditions.

The present study conducted among 98 clinically suspected pityriasis versicolor cases revealed prevalence of four malassezia species whereas in various studies most commonly isolated species are seven in number. Chowdhury et al,[9] isolated 12 malassezia species of which M. globosa, M. sympodialis, M. furfur were of clinically significant. Present study also documented this fact.It revealed M. furfur to be the most prevalent species among the 88 culture positive cases. It is supported by a few studies conducted under the tropical climate[19,20]. Next in frequency was M. globosa followed by M. restricta and M. sympodialis. This findings are contradictory to the studies by Aspiroz et al[21], Nakabayashi et al[7] and Crespo Erchiga et al[22] who isolated M. globosa as the most common species at the frequencies of $58.2 \%, 55 \%$ and $55 \%$ respectively.

In two similar studies have been done in South India by Kindo et al,[18]revealed that $\mathrm{M}$. sympodialis is the commonest agent (58.3\%) followed by M. globosa (39.6\%).

Another study from North-Central India, conducted by Dutta et al,[23]revealed that $54 \%$ of isolates belonged to M.globosa and the next common species being M.furfur( $30 \%)$.

Of the previous 17 studies, 11 showed M. globosa to be the most common species isolated. Its pathogenecity might be explained by high lipolytic activity[22]. A few studies have found $M$. sympodialis to be the most common species [18].

The differences in frequencies of malassezia species among different studies may be attributed to different culture media(MDA/LNA) used and perhaps to ethnic and geographic factors[20].

Rahul et al,[9] found no definite relationship of species and the pigmentary changes produced. This supports the present study finding where only 5 cases presented with hyperpigmented lesions. Along with this as the predominant isolate in both hypo- and hyper-pigmented isolates was M.furfur,probably no relationship exists.

\section{Conclusion:}

Thus malassezia species were found to be the causative organism of pityriasis versicolor. Both hypo-or hyper-pigmented macular rashes were seems to be clinically significant.

The lesion is most prevalent in the age group of $15-25 \mathrm{yrs}$. The present study suggests M.furfur to be the most prevalent species, followed by M. globosa, M. restricta and M. sympodialis. Pityriasis versicolor is a superficial skin disease involving upto the stratum corneum layer of the skin and the sebaceous glands. The main complain of the patients being cosmetic and itchiness on profuse sweating.

\section{Acknowledgement:}

I express my gratitude to Dr. Manas Bandopadhyay,who was my guide in performing this research work as dissertation during my post graduate trainee period.I have been supported by the Microbiology laboratory of RGKar Medical \& Hospital where I completed my MD in Microbiology.The expenses of the research work was borne partly by government and partly by myself.

\section{References:}

[1]. Bigby, M. and Casulo, C. Pityriasis versicolor....In:Burns, T., Breathnach, S., Cox, N. and Griffiths, C. (Eds.) Rook's textbook of dermatology. Oxford: Blackwell Science.2008; 8th edn.vol 2:36.10-36.14.

[2]. Burkhart, C.G. and Gottwald, L. Tinea versicolor. eMedicine. WebMD.2010.

[3]. Midgley, G., Gue'ho, E. and Guillot, J.Diseases caused by Malassezia In: Topley and Wilson_s Microbiology and Microbial Infections (Ajello, L. and Hay, R.J., Eds.).1998; Vol. 4, pp. 201-211.

[4]. Crespo-Erchiga, V. and Gue'ho, E. Superficial diseases caused by Malassezia species. In: Topley and Wilson_s Microbiology and Microbial Infections, Mycology (Hay, R. and Merz,W., Eds.), Arnold, London, UK.2005 .Vol. 5, 10th edn.

[5]. Midgley G. The lipophilic yeasts: State of the art and prospects. Med Mycol 2000; 38 Suppl 1:9-16.

[6]. Crespo Erchiga V, Ojeda Martos A,Vera Casano A, Crespo Erchiga A, Sanchez Fajardo F. Malassezia globosa as the causative agent of Pityriasis Versicolor. Br J Dermatol .2000: 143: 799-803.

[7]. Nakabayashi A,Sei Y, Guillot J. Identification of Malassezia sp isolated from patients with seborrhoeic dermatitis, Atopic dermatitis, Pityriasis Versicolor and normal subjects. Med Mycol . 2000,38:337-341.

[8]. Gupta AK, Kohli Y, Li A, Faergemann J, Summerbell RC. In vitro susceptibility of the seven Malassezia species to ketoconazole, voriconazole, itraconazole and terbinafine. Br J Dermatol 2000; 142:758-765.

[9]. Choudhary R, Singh S, Banerjee T, Tilak R. Prevalene of different Malassezia species in Pityriasis Versicolor in Central India. Indian Journal of Dermatology Venereology Leprology. 2010; Vol 76(2): 159-164.

[10]. Shams M, Rasaee MJ, Moosvi M,et al: Identification of Malassezia species in patients with Pityriasis Versicolor a study in Tehran. Iranian Biomed J. 2001; 5: 121-126. 
[11]. Sugita T, Takashima M, Kodama M, Tsuboi R, Nishikawa A. Description of a new yeast species, Malassezia japonica , and its detection in patients with atopic dermatitis and healthy subjects. J. Clin. Microbiol. 2003;41: 4695-4699.

[12]. El-Hefnawi H, El-Gothamy F, Regai M. Studies on Pityriasis Versicolor in Egypt:I. Incidence. Mycosen.1971; 14(5): $225-231$.

[13]. Michalowski R, Rodziewicz H. Pityriasis Versicolor in children. Brit J Dermatol. 1963; 75: 397-400.

[14]. Miskeen AK, Kelkar SS, Shroff J. Pityriasis Versicolor in children. Indian Journal of Dermatology Venereology Leprology. 1984;50: 144-146.

[15]. Marples MJ. The incidence of certain skin disease in Western Samoa: A preliminary survey. Trans Roy Soc Trop Med Hyg. 1950; 44: 419.

[16]. Smith EB, Gellerman GL. Tinea versicolor in infancy. Arch Derm. 1966; 93: 362-363.

[17]. El-Gothamy M.G. Zenab. A review of Pityriasis Versicolor J Egypt Wom Dermatol Soc. 2004: Vol 1(2): 36-43.

[18]. Kindo AJ, Sophia SKC, Kalyani J, Anandan S. Identification of Malassezia species. IJMM, 2004;22(3) : 179-181.

[19]. Krisanty RI, Bramono K, Made Wisnu I. Identification of Malassezia species from Pityriasis Versicolor in Indonesia and its relationship with clinical charecteristics. Mycoses. 2009;52: 257-262.

[20]. Miranda KC, de Araeyo CR, Soares AJ, de Aquino Lemos J,Souza LK, do Rosario Rodrignes, Silva M. Identification of Malassezia species in patients with Pityriasis Versicolor in Gviania-GO. Rev. Soc Bras Med Trop. 2006; 39: 582-583.

[21]. Aspiroz C, Ara M, Varea M, Rezusta A, Rubio C. Isolation of Malassezia globosa and Malassezia sympodialis from patients with Pityriasis Versicolor in Spain. Mycopathologia. 2002; 154:111-117.

[22]. Crespo Erchiga V, Ojeda Martos A, Vera Casano A, Crespo Erchiga A, Sanchez Fazardo F, Guheo E. Mycology of Pityriasis versicolor. J Mycol Med. 1999;9: 143-148.

[23]. Dutta S, Bajaj AK, Basu S, Dikshit A. Pityriasis Versicolor: Socio-economic and clinico-mycologic study in India. Int J Dermatol.2002; 41:823-824. 